\title{
Epitaxial 2D PbS Nanoplates Arrays with Highly Efficient Infrared Response
}

\author{
Yao Wen, Qisheng Wang, Lei Yin, Qi Liu, Feng Wang, Fengmei Wang, Zhenxing Wang, \\ Kaili Liu, Kai Xu, Yun Huang, Tofik Ahmed Shifa, Chao Jiang,* Jie Xiong,* and Jun He*
}

\begin{abstract}
2D materials such as graphene, ${ }^{[1]} \mathrm{MoS}_{2},{ }^{[2]} \mathrm{Bi}_{2} \mathrm{Se}_{3}$, and $\mathrm{Bi}_{2} \mathrm{Te}_{3}{ }^{[3]}$ are now one of the most intensive studies owing to their unconventional surface-related physical, optical, and electronic properties. They are promising candidates in next-generation electronics and optoelectronics devices due to their fascinating properties including high electron or hole mobility, ${ }^{[1,4]}$ quantum Hall effects, ${ }^{[5]}$ superconductivity, ${ }^{[6]}$ quantum anomalous Hall effect, ${ }^{[7]}$ extraordinary thermal conduction, ${ }^{[8]}$ and great potential applications in sensing, ${ }^{, 9]}$ transistors, ${ }^{[10]}$ biomedicine, ${ }^{[11]}$ photodetectors. ${ }^{[12]}$ Benefiting from planar geometry, 2D nanostructures possess great advantages over the bulk materials for flexible devices and exhibit high compatibility with traditional microfabrication techniques. Furthermore, 2D nanostructures show surprising properties due to strong quantum confinement effect and ultrahigh specific surface area. However, the current research is mainly limited to the field of 2D layered
\end{abstract}

\footnotetext{
Y. Wen, L. Yin, F. Wang, F. Wang, Prof. Z. Wang, K. Liu, K. Xu, Y. Huang, T. A. Shifa, Prof. C. Jiang, Prof. J. He CAS Center for Excellence in Nanoscience

National Center for Nanoscience and Technology

Beijing 100190, China

E-mail: jiangch@nanoctr.cn; hej@nanoctr.cn

Y. Wen, Prof. C. Jiang

CAS Key Laboratory for Standardization and Measurement for

Nanotechnology

National Center for Nanoscience and Technology

Beijing 100190, China

Y. Wen, L. Yin, F. Wang, F. Wang, Prof. Z. Wang, K. Liu, K. Xu, Y. Huang,

T. A. Shifa

University of Chinese Academy of Sciences

Beijing 100049, China

Dr. Q. Wang

Department of Electrical and Computer Engineering

National University of Singapore

Singapore 117576, Singapore

L. Yin, F. Wang, F. Wang, Prof. Z. Wang, K. Liu, K. Xu, Y. Huang,

T. A. Shifa, Prof. J. He

CAS Key Laboratory of Nanosystem and Hierarchical Fabrication

National Center for Nanoscience and Technology

Beijing 100190, China

Prof. Q. Liu

Key Laboratory of Microelectronic Devices and Integrated Technology Institute of Microelectronics

Chinese Academy of Sciences

Beijing 100029, China

Prof. J. Xiong

State Key Laboratory of Electronic Thin Film and Integrated Devices

University of Electronic Science and Technology of China

Chengdu 610054, China

E-mail: jiexiong@uestc.edu.cn
}

DOI: 10.1002/adma.201602481 materials such as graphene and $\mathrm{MoS}_{2}$ as mentioned at the beginning. The exploration of $2 \mathrm{D}$ nonlayered materials will bring us exotic electronic and optoelectronic properties beyond layered materials due to novel electronic structure of nonlayered materials. As demonstrated in our previous work that 2D $\mathrm{Pb}_{1-x} \mathrm{Sn}_{x} \mathrm{Se}$ nanoplates exhibit broad and sensitive infrared response due to its direct narrow bandgap and high absorption coefficient. Recently, Zhang et al. demonstrated the controlled epitaxial growth of 1D CdSe nanorods array on 2D CdS nanoplates. The nanorod arrays were grown on the selective facets of the hexagonal nanoplates via seed engineering. This hierarchical 1D/2D nonlayered nanostructures have great potential in a variety of fields such as energy conversion, electronics. ${ }^{[13]}$ Xie et al. showed the ultrathin nanosheets of the Mn-doped $\mathrm{CoSe}_{2}$ exhibited useful hydrogen evolution reaction properties, which provided a new way for newly highly efficient catalysts. ${ }^{[14]}$ Therefore, the synthesis of $2 \mathrm{D}$ nonlayered materials is a pressing topic for their potential applications.

Precise control on the growth site of nanomaterial has been an essential and challenging topic. Significantly, individual crystal has shown great superiority in some applications, which require minimal cross-talk between neighboring devices including active matrix displays and sensor arrays. ${ }^{[15]}$ Apparently, individual crystal arrays are critical in preparing practical devices with high performance and density. Throughout the history of van der Waals epitaxial 2D nanostructures arrays, it has achieved great progress but limited in layered nanostructures such as $\mathrm{GaSe}^{[16]} \mathrm{Bi}_{2} \mathrm{Se}_{3}, \mathrm{Bi}_{2} \mathrm{Te}_{3}, \mathrm{In}_{2} \mathrm{Se}_{3}$, SnSe, ${ }^{[17,18]}$ lead halide $\left(\mathrm{PbI}_{2}\right),{ }^{[19,20]}$ etc. Our group has made a big breakthrough in the growth and device applications of 2D nonlayered semiconductor such as van der Waals epitaxial $2 \mathrm{D} \mathrm{Te},{ }^{[21]} \mathrm{Pb}_{1-x} \mathrm{Sn}_{x} \mathrm{Se}^{[22,23]}$ nanoplates on mica or h-BN. However, patterning the 2D nonlayered semiconductor array is still a big challenge due to complicated growth dynamics of 2D nonlayered materials. Layered materials hold the intrinsic driving force for 2D anisotropic growth while 2D nonlayered materials lack this due to its 3D isotropic crystal structure. And crystal structure of layered materials matches well with layered epitaxial substrate (for example, $\mathrm{MoS}_{2}$ shares the same hexagonal symmetry with growth substrate such as graphene, mica). However, nonlayered semiconductor exhibits completely different crystal orientation with layered epitaxial substrates.

Owing to the rapid deterioration of silicon photosensitive properties beyond $1100 \mathrm{~nm}$, the narrow bandgap semiconductor has emerged such as $\operatorname{InAs}_{1-x} \mathrm{Sb}_{x}, \mathrm{~Pb}_{1-x} \mathrm{Sn}_{x} \mathrm{Te}$, $\mathrm{Hg}_{1-x} \mathrm{Cd}_{x} \mathrm{Te}, \mathrm{PbSe}, \mathrm{PbTe}$, InAs, InSb, PbS.[24,25] These materials possess outstanding optoelectronics properties including high electron mobility, high optical absorption coefficient, and 
low thermal generation rate. As a typical example, $\mathrm{Hg}_{1-x} \mathrm{Cd}_{x} \mathrm{Te}$ has shown great superiority in infrared (IR) radiation detectors owing to easy bandgap tailoring, multicolor detectors. However, $\mathrm{Hg}_{1-x} \mathrm{Cd}_{x} \mathrm{Te}$ suffers nonuniformity over large area, surface instability, and complicated growth methods. ${ }^{[25]}$ In contrast, $\mathrm{PbS}$ is easier to prepare and more stable with the direct narrow bandgap $(0.4 \mathrm{eV}) .{ }^{[26]}$ Significantly, benefiting from the broad spectral detection from the visible to mid-IR region, $\mathrm{PbS}$ shows many applications in remote sensing and environmental monitoring, ${ }^{[27]}$ biological imaging (transparent tissue windows from 800 to $1100 \mathrm{~nm}$ ), telecommunications (1300 to $1600 \mathrm{~nm}$ ), thermal imaging (1500 $\mathrm{nm}$ and beyond), thermal photovoltaics (>1900 nm). ${ }^{[28]}$ Moreover, according theoretical predication, few-layer $\mathrm{PbX}(\mathrm{X}=\mathrm{S}, \mathrm{Se}, \mathrm{Te})$ has an extremely high electron or hole carrier mobility. ${ }^{[29]}$ Interestingly, a recent work displays the normalized carrier multiplication efficiency is higher in 2D PbS nanosheets compared with its 0D quantum dots, 1D nanorods, and 3D bulk state. ${ }^{[30]}$

Herein, we report epitaxial growth of ultrathin, highly efficient 2D nonlayered PbS nanoplates via van der Waals epitaxy. The nanoplates with thickness ranging from 5 to $35 \mathrm{~nm}$ was synthesized on graphite. The devices displayed outstanding photoresponsivity, detectivity, switching time and photogain values as high as $1621 \mathrm{~A} \mathrm{~W}^{-1}, 1.72 \times 10^{11}$ Jones, $0.3 \mathrm{~s}$ and 2512 , respectively, which were even comparable with previously hybrid heterostructures. ${ }^{[31-35]}$ Moreover, we achieved precise control over the orientation, position of $\mathrm{PbS}$ nanoplates on the flexible mica substrate. To the best of our knowledge, we for the first time realize the controllable synthesis of inorganic 2D nonlayered semiconductor arrays via van der Waals epitaxy, which is significant for exploring integrated infrared sensors.

Figure 1a shows schematic illustration of $\mathrm{PbS}$ crystal structure. Remarkably, unlike 2D layered nanostructures with strong planar covalent bonding in lateral dimension and weak van der Waals interaction between two adjacent layers, cubicsymmetry $\mathrm{PbS}$ is of $3 \mathrm{D}$ bonding with isotropic structure. The 2D $\mathrm{PbS}$ nanoplates were grown on layered mica and graphite. Figure $1 \mathrm{~b}$ presents one of typical samples on graphite. The $\mathrm{PbS}$ powders were used as the precursor in a low-pressure chemical vapor deposition (LPCVD) systems. Interestingly, $\mathrm{PbS}$ nanoplates selectively grew on graphite, as shown in Figure 1b and Figure S1 (Supporting Information). The selective growth behavior could be explained by the classical nucleation kinetics as discussed in previous reports. ${ }^{[34,36]}$ In our case, graphite without surface dangling bonds shows low surface energy $\left(\gamma_{\text {graphite }} \approx 70-80 \mathrm{~mJ} \mathrm{~m}{ }^{-2}\right),{ }^{[37]}$ which is in favor of the lateral growth of $\mathrm{PbS}$ nanoplates along surface of graphite. In contrast, owing to the larger surface energy of $\mathrm{SiO}_{2}\left(\gamma_{\mathrm{SiO} 2} \approx\right.$ $\left.300-400 \mathrm{~mJ} \mathrm{~m}{ }^{-2}\right),{ }^{[34]}$ the lateral growth of the $\mathrm{PbS}$ crystals on $\mathrm{SiO}_{2} / \mathrm{Si}$ must be overcome large free energy barrier. ${ }^{[34]}$ In addition, owing to the strong interaction between dangling bonds on the surface of $3 \mathrm{D}$ bonded $\mathrm{SiO}_{2}$ and adatoms, the migration coefficient on $\mathrm{SiO}_{2}$ is far lower than that on atomically smooth graphite surface. ${ }^{[22]}$ Consequently, compared to $\mathrm{SiO}_{2}$ surface, the lower surface energy and larger migration coefficient are the two key factors that $\mathrm{PbS}$ nanoplates selectively grew on graphite under optimized reaction parameters. Van der Waals epitaxy features weak van der Waals interaction between overlayer and substrate. It requires that either of overlayer or substrate is free of surface dangling bonds. Here we utilize mica and graphite as the growth substrate is based on the fact that they are both layered materials, the freshly exfoliated surfaces of which are highly smooth and chemically passivated. In addition, the van der Waals epitaxy allows a larger lattice mismatch between overlayer and substrate. That we see $\mathrm{PbS}$ nanoplates selectively grow on graphite but not $\mathrm{SiO}_{2}$ substrates under the same

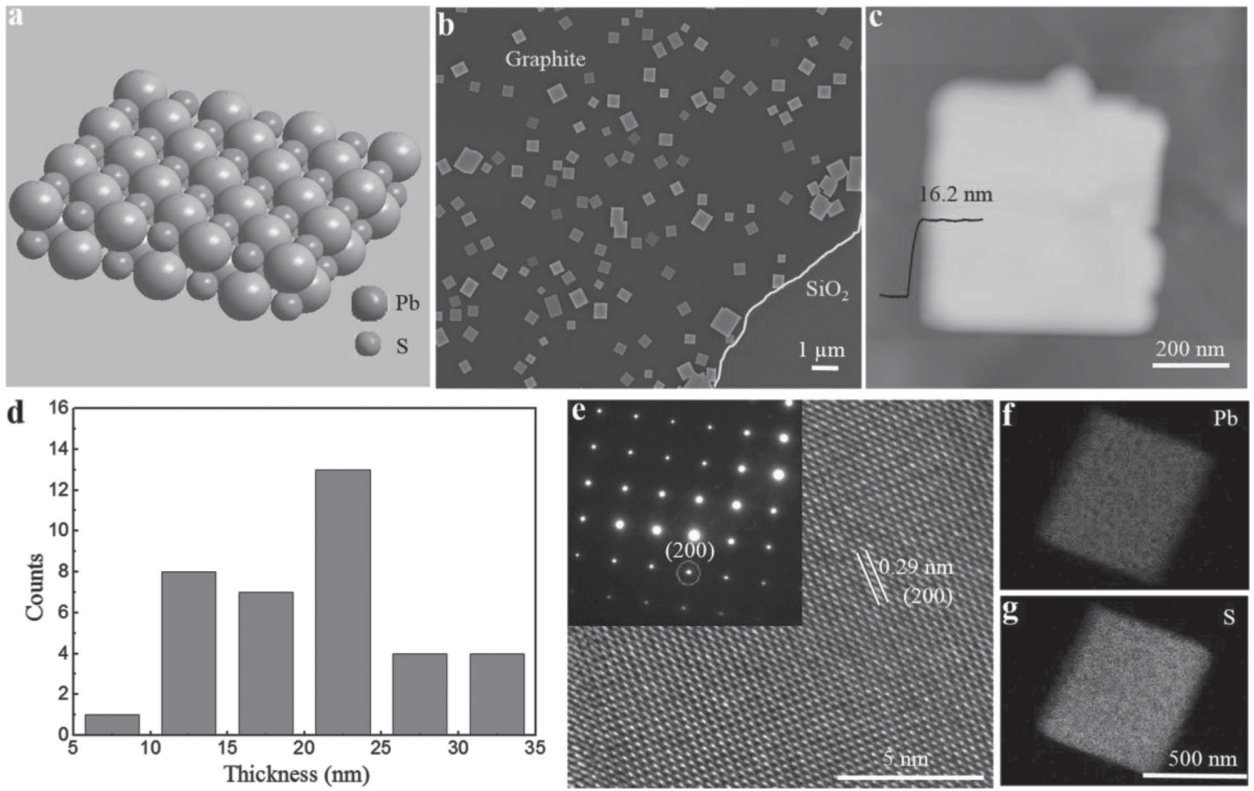

Figure 1. Morphology of vdWE PbS nanoplates. a) Schematic illustrations of the PbS with cubic crystal structure. b) SEM image of square PbS nanoplates on graphite. c) AFM image of one square nanoplate with thickness of $16.2 \mathrm{~nm}$. d) The histogram statistics of nanoplates with thickness ranging from 5 to $35 \mathrm{~nm}$. e) HRTEM image with clear crystal fringes of (200) planes with corresponding SAED as the inset. f,g) TEM-EDX mapping of PbS nanoplate. 
growth conditions, which would be understood by the fact, as a conventional growth mode, large lattice mismatch between $\mathrm{PbS}$ (cubic) and $\mathrm{SiO}_{2}$ (amorphous) hinders the epitaxy of $\mathrm{PbS}$ nanoplates on $\mathrm{SiO}_{2}$. Figure 1c presents the atomic force microscopy (AFM) image of one square nanoplate with thickness of $16.2 \mathrm{~nm}$. The thickness of $\mathrm{PbS}$ nanoplates is critical for the photoelectronic properties. The increase of nanoplates thickness will enhance the light harvesting. However, since photocurrent results from transport of photoexcited carriers, the signal-to-noise ratio likely decreases as the thickness of $\mathrm{PbS}$ nanoplates excesses the critical value. The numbers of photoexcited carriers are limited by the absorption coefficient. As the thickness of $\mathrm{PbS}$ nanoplates excesses the critical value, the underlayer of $\mathrm{PbS}$ nanoplate which has not been irradiated by laser will cause extra dark current. The thickness can be controlled by manipulating reaction time, carrier gas flow rate and temperature in the CVD process. The root-mean-square (RMS) roughness of nanoplates is less than $0.1 \mathrm{~nm}$, which unambiguously verifies the atomically smooth surface of $\mathrm{PbS}$ nanoplate. The histogram statistics of thickness demonstrate that the thickness of nanoplates ranges from 5 to $35 \mathrm{~nm}$, as shown in Figure 1d. The original AFM image presents in Figure S2 (Supporting Information). In order to investigate the crystallinity of the nanoplates, transmission electron microscopy (TEM) was employed. Figure 1e indicates the high-resolution TEM (HRTEM) images with clear crystal fringes of (200) planes, which demonstrates that the PbS nanoplate is well-defined single crystalline. The lattice constant of (200) planes is $0.29 \mathrm{~nm}$. The top surfaces of the square nanoplate can be assigned to (002). As PbS possesses highly symmetry cubic structure, we can identify the edge orientation of $\mathrm{PbS}$ square nanoplates by direct TEM observation. As presented in inset of Figure 1e, the square SAED pattern reveals (200) planes of $\mathrm{PbS}$ nanoplates, indicating the edge oriented along [200] direction. The cubic crystal structure of $\mathrm{PbS}$ is confirmed by selected area electron diffraction (SAED), wherein the perfect cubic pattern clearly reveals the cubic symmetry and high crystallinity of the nanoplates. TEM-EDX elemental mapping (Figure 1f,g) indicates that $\mathrm{Pb}$ and $\mathrm{S}$ distribute uniformly in the entire nanoplate without detectable phase separation. An elemental quantitative analysis of TEM-EDX shows the atomic percentage ratio of $\mathrm{Pb}$ and $\mathrm{S}$ to be about 1:1.03, indicating ideal chemical stoichiometry.

The inset of Figure 2a shows the SEM images of the single $\mathrm{PbS}$ nanoplate device, which is transferred onto clean $\mathrm{SiO}_{2} /$ Si substrate from mica. The infrared photodetectors based on $\mathrm{PbS}$ nanoplates were fabricated on $\mathrm{SiO}_{2} / \mathrm{Si}$ substrate by standard electron-beam lithography (EBL). Figure 2a presents the electrical transport characteristic of single $\mathrm{PbS}$ nanoplate. The obvious photoresponse can be detected with a series of wavelengths 800, 1064, $1340 \mathrm{~nm}$ lasers with effective intensity of 17.9, 15.1, $9.2 \mathrm{pW}$, respectively. The effective intensity means the laser power on the device channel. The effective irradiated area is about $0.69 \mu \mathrm{m}^{2}$. In addition, the linear plot of current $(I)$-voltage $(V)$ curve exhibits the Ohmic contact in our device. Photoresponse of single $\mathrm{PbS}$ nanoplate at $800 \mathrm{~nm}$ laser is further executed, as shown in Figure $2 \mathrm{~b}$. The $I_{\mathrm{ph}}\left(I_{\mathrm{ph}}=I_{\text {illumination }}-I_{\text {dark }}\right)$ increases from 0.2 to $2.47 \mu \mathrm{A}$ as the effective intensity varies from 0.6 to $60.4 \mathrm{pW}$, exhibiting the high detection sensitivity of our device. The photocurrent is linearly proportional to the incident light power $I_{\mathrm{ph}} \approx P \beta$ with $\beta=0.5$, as shown in Figure 2c. ${ }^{[38]}$ The linear relationship indicates that the photocurrent is determined by photoconductive mechanism. The value of $\beta$ is expected to be 1 in ideal case. The recombination of the photogenerated free carriers and charge trapping
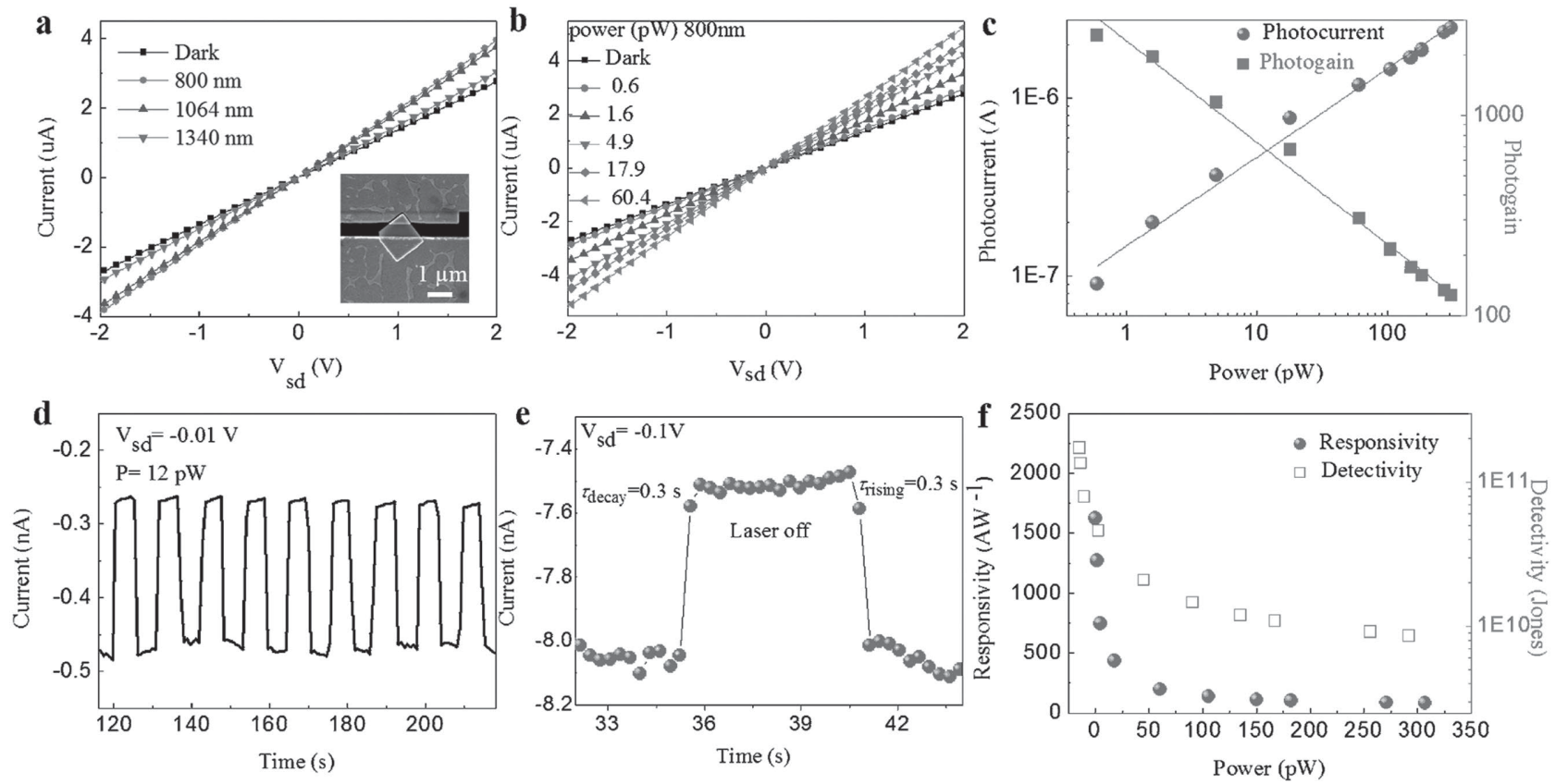

Figure 2. Infrared response of the PbS nanoplates device. a) Photoresponse of single PbS nanoplate with irradiation of 800 , 1064 , 1340 nm laser. b) Photoresponse of the nanoplate with irradiation of $800 \mathrm{~nm}$ laser. c) The plot of photogain (G) and photocurrent versus laser power at $800 \mathrm{~nm}$. d) Time-dependent photoresponse of PbS nanoplate device at $800 \mathrm{~nm}$. e) A separated response and reset cycle: $\tau_{\text {rising }}=\tau_{\text {decay }}=0.3 \mathrm{~s}$, which corresponds to $100 \%$ rising and decay. f) The plots of responsivity and detectivity versus laser power. 
Table 1. Comparison of optoelectronic parameters with hybrid heterostructures.

\begin{tabular}{lcccc}
\hline & Wavelength & Responsivity & Response time $^{\text {a) }}$ & Refs. \\
\hline PbS quantum dots-graphene & $600 \mathrm{~nm}$ & $5 \times 10^{7}\left[\mathrm{~A} \mathrm{~W}^{-1}\right]$ & $\tau_{\text {rising }}=\tau_{\text {decay }}=2 \mathrm{~s}$ & {$[31]$} \\
PbS quantum dots-graphene (CVD-grown) & $895 \mathrm{~nm}$ & $1 \times 10^{7}\left[\mathrm{~A} \mathrm{~W}^{-1}\right]$ & $\tau_{\text {rising }}=0.3 \mathrm{~s}$ & {$[32]$} \\
& & & $\tau_{\text {decay }}=1.7 \mathrm{~s}$ & \\
PbSe quantum dots-MoS 2 & $>1200 \mathrm{~nm}$ & $1.9\left[\mu \mathrm{A} \mathrm{W}{ }^{-1}\right]$ & $\tau_{\text {rising }}=0.25 \mathrm{~s}$ & {$[35]$} \\
& & & $\tau_{\text {decay }}=0.43 \mathrm{~s}$ & \\
CdS nanoplates-MoS 2 & $610 \mathrm{~nm}$ & $3.91\left[\mathrm{~A} \mathrm{~W}^{-1}\right]$ & $\tau_{\text {rising }}>0.1 \mathrm{~s}$ & {$[34]$} \\
PbS quantum dots-MoS $\mathrm{MoS}_{2}$ & $635 \mathrm{~nm}$ & $6 \times 10^{5}\left[\mathrm{~A} \mathrm{~W}^{-1}\right]$ & $\tau_{\text {decay }}=0.35 \mathrm{~s}$ & {$[33]$} \\
PbS nanoplates & $800 \mathrm{~nm}$ & $1621\left[\mathrm{~A} \mathrm{~W}^{-1}\right]$ & $\tau_{\text {rising }}=\tau_{\text {decay }}=0.3 \mathrm{~s}$ & This work \\
\hline
\end{tabular}

a) $\tau_{\text {rising }}$ and $\tau_{\text {decay }}$ correspond to $100 \%$ rising and decay, respectively.

could be responsible for the loss of photocurrent. ${ }^{[38]}$ In the ideal photoconductive detector, $I_{\mathrm{ph}}$ is proportional to $P \beta$ with power factor $\beta$ of 1 . In our case, $\beta$ equals to 0.5 , indicating the loss of photogenerated electron-hole pairs. For example, the vacancies in $\mathrm{PbS}$ nanoplates form the localized states, which can trap the photoexcited carriers. And the unsaturated bonds on surface or interface of $\mathrm{PbS}$ nanoplates also behave as trap states centers, which capture photoexcited carriers. In addition, nonradiative recombination of electron-holes pair through phonon coupling is also responsible for loss of photo-excited carriers. Photogain $(G)$ is given by the formula: $G=\left(I_{\mathrm{ph}} / q\right) /(\mathrm{PS} / h v)=$ $h \nu R \lambda / q$, where $q$ is the electron charge, $h$ is Planck's constant, $v$ is the light frequency, and $R \lambda$ is responsivity. ${ }^{[39]}$ The device exhibits great photogain as high as 2512, as shown in Figure 2c. Figure $2 \mathrm{~d}$ exhibits time-resolved photoresponse at wavelength $800 \mathrm{~nm}$ with light power of $12 \mathrm{pW}$. The device is highly stable and reversible, which indicates almost the same level with light off. The magnified photoresponse cycle (Figure 2e) exhibits $\tau_{\text {rising }}=\tau_{\text {decay }}=0.3 \mathrm{~s}$ corresponding to $100 \%$ rising and decay, which is even comparable with previously hybrid heterostructures, as shown in Table $1 .^{[31-33,35]}$ The responsivity $(R)$ is defined by $I_{\mathrm{ph}} / \mathrm{PA}, A$ is the irradiated area, $P$ is the light density. We extract the responsivity $(R)$, which maintains in the range of 81 to $1621 \mathrm{~A} \mathrm{~W}^{-1}$ as effective intensity distributing between 306.7 and $0.6 \mathrm{pW}$ as shown in Figure 2f. The responsivity represents the ability of light harvesting. Significantly, when effective intensity drops to $0.6 \mathrm{pW}, R$ dramatically increases to $1621 \mathrm{~A} \mathrm{~W}^{-1}$ which is even better than previously hybrid heterostructures, ${ }^{[34,35]}$ as shown in Table 1 . Detectivity $\left(D^{*}\right)$ can be expressed as $D^{*}=I_{\mathrm{ph}} \mathrm{A}^{1 / 2} / \mathrm{PA}\left(2 q I_{\text {dark }}\right)^{1 / 2}$. In our case, $D^{*}$ values of $\approx 1.72 \times$ $10^{11}$ Jones is achieved at $P=0.6 \mathrm{pW}$, as shown in Figure $2 \mathrm{f}$.

Significantly, the practical infrared detectors require minimal cross-talk between neighboring devices. ${ }^{[15]}$ Therefore, individual crystal has shown great superiority than thin film. Here, we precisely controlled the orientation, position of the cubic $\mathrm{PbS}$ nanoplates on the flexible fluorophlogopite $\left[\mathrm{KMg}_{3}\left(\mathrm{AlSi}_{3} \mathrm{O}_{10}\right) \mathrm{F}_{2}\right]$ mica substrate. Mica with a pseudohexagonal $\mathrm{Z}_{2} \mathrm{O}_{5}(\mathrm{Z}=\mathrm{Si}, \mathrm{Al})$ layered structure is an ideal chemically inert and atomically planar surface for vdWE. Orientationcontrolled growth of PbS nanoplates is crucial for next-generation integrated optoelectronic circuits. Figure 3a exhibits optical microscope (OM) images with orientation-controlled $18 \times 16$ arrays of square $\mathrm{PbS}$ nanoplates on mica. The

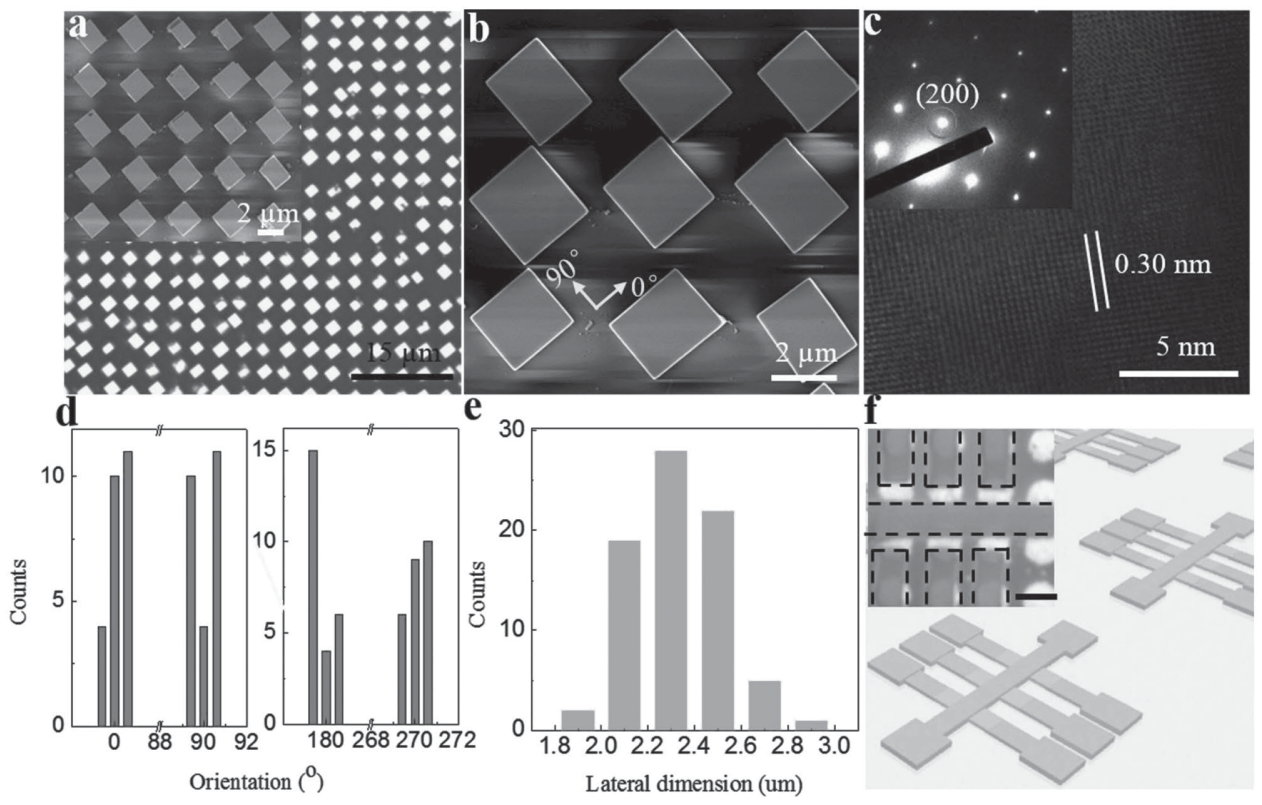

Figure 3. Morphology of 2D PbS nanoplates arrays. a) OM image of orientation-controlled $18 \times 16 \mathrm{PbS}$ nanoplates arrays on mica. The inset shows SEM image of $5 \times 5$ PbS arrays. b) Magnified SEM image of $3 \times 3$ PbS arrays. c) HRTEM image of the single PbS with corresponding SAED as the inset. d) Histogram of the orientation distribution of $\mathrm{PbS}$ arrays obtained from 50 nanoplates. The edges of square nanoplates on mica are oriented predominantly at multiples of $90^{\circ}$. e) Histogram of the lateral dimension of $\mathrm{PbS}$ arrays obtained from 77 nanoplates. f) Schematic illustration of PbS integrated device arrays, top insert: OM image of PbS-integrated device arrays, the scale bar is $2 \mu \mathrm{m}$. 
well-ordered $5 \times 5$ and $3 \times 3$ arrays of square $\mathrm{PbS}$ are also presented in the SEM images (the inset of Figure 3a,b). HRTEM and corresponding SAED images of the individual $\mathrm{PbS}$ nanoplate reveal that nanoplates are well-defined single-crystalline phase with clear crystal fringes of (200) planes, as shown in Figure 3c. We have executed the XRD on PbS nanoplates arrays. As shown in Figure S7a (Supporting Information), the peaks of (111), (200), (220), (222), (400) are consistent with standard JCPDS card (PDF\#77-0244), indicating the highly single-crystalline of $\mathrm{PbS}$ nanoplates. We have performed the UV-VIS-NIR spectroscopy on $\mathrm{PbS}$ nanoplates arrays. As we can see from Figure S7b in the Supporting Information, it exhibits a broad absorption from 200 to $840 \mathrm{~nm}$, which matches well with its narrow bandgap $(0.4 \mathrm{eV})$. The cutoff of spectra absorption (theoretically $3100 \mathrm{~nm}$ ) has not been observed due to limited detection range $(200-840 \mathrm{~nm})$ of our UV-vis-infrared spectroscopy. From the absorption spectra, we can see $800 \mathrm{~nm}$ lasers are suitable for photoresponse measurements. To quantitatively describe the orientation-controlled $\mathrm{PbS}$ nanoplates, well-ordered arrays with 50 nanoplates are analyzed as shown in Figure 3d. The edges of square nanoplates on mica are oriented predominantly at multiples of $90^{\circ}$. Compared with the random orientation of the $\mathrm{PbS}$ nanoplates grown on graphite, the orientationcontrolled growth of nanoplates indicates that the epitaxial nature between mica and $\mathrm{PbS}$ is crucial for well-ordered arrays. ${ }^{[3,16-18,34,40,41]}$ Although the mechanism merits further investigation, we propose one possibility to elucidate the epitaxial relation between $\mathrm{PbS}$ nanoplates and mica. The lattice constant of mica corresponds to $a=0.53 \mathrm{~nm}, b=0.92 \mathrm{~nm}$, and $c=1.01 \mathrm{~nm} \cdot{ }^{[40]}$ Noticeably, the surfaces of mica $(00 \mathrm{n})$ are predominantly occupied by $\mathrm{K}$ atoms, as previously reported. ${ }^{[40]}$ As shown in Figure S3 (Supporting Information), the hexagonal $\mathrm{K}$ atom pattern of mica (00n) surfaces brings about four equivalent epitaxial relations with $\mathrm{PbS}$ final exposed four $\{200\}$ crystal surfaces, which results in four orientations $\left(0^{\circ}, 90^{\circ}, 180^{\circ}\right.$, and $\left.270^{\circ}\right)$ of $\mathrm{PbS}$ nanoplates arrays. The lattice mismatch between $\{100\}$ lattice planes $(0.59 \mathrm{~nm})$ of PbS (JCPDS card NO. 78-1897) and the K atoms
$(0.53 \mathrm{~nm})$ is $11 \%$. Owing to the weak van der Waals interaction at the interface, van der Waals epitaxial allows large lattice mismatch, such as $32 \%$ for CdS and $\mathrm{MoS}_{2}, 12 \%$ for $\mathrm{PbSe}$ and $\mathrm{MoS}_{2}, 6 \%$ for $\mathrm{Pb}_{1-x} \mathrm{Sn}_{x}$ Te and mica. ${ }^{[34,35,40]}$ The ordered growth of $\mathrm{PbS}$ nanoplates array further confirms the nature of van der Waals epitaxy as PbS shows a lattice mismatch with mica as large as 11\%. A high-quality interface between $\mathrm{PbS}$ nanoplates and substrate is expected although the interface lattice orientation has not been directly observed due to the difficulties in fabricating the cross-sectional sample. In the van der Waals epitaxy, the weak interaction in the interface allows strain relaxation, which is less possible in conventional epitaxial growth. Statistics of SEM images give the lateral dimension distribution ranging from 1.8 to $3 \mu \mathrm{m}$, which exhibits the highly controlled lateral sizes of nanoplates, as shown in Figure 3e. Figure S4 (Supporting Information) exhibits the AFM image and height profile (white dashed line) of $\mathrm{PbS}$ nanoplates, which shows flat surfaces with thickness ranging from 100 to $200 \mathrm{~nm}$. Figure $3 \mathrm{f}$ exhibits the schematic illustration of $\mathrm{PbS}$-integrated device arrays. The inset shows the $3 \times 2$ device arrays on mica.

We realize epitaxial $\mathrm{PbS}$ nanopliates array by utilizing $\mathrm{NaCl}$ aqueous solution to define the selectively van der Waals epitaxy of $\mathrm{PbS}$ nanoplates. First, mica was intaglioed by $\mathrm{NaCl}$ aqueous solution using a polydimethylsiloxane (PDMS) stamp, ${ }^{[18]}$ as shown in Figure 4a. Then, mica with $\mathrm{NaCl}$ aqueous solution were heated to $150{ }^{\circ} \mathrm{C}$ using a heating belt. Finally, the mica surface modified with periodic arrays was released from the PDMS stamp. Benefiting from the hydrophilic surface of mica, uniform patterns can be achieved, as shown in Figure S5 (Supporting Information). After printing and drying, selectivearea van der Waals epitaxy of $\mathrm{PbS}$ crystals was employed. ${ }^{[1]}$ In order to reveal the growing mechanism of $\mathrm{PbS}$ nanoplates arrays, the surface morphology of the $\mathrm{PbS}$ nanoplates was investigated by SEM under different reaction times. Interesting, a large amount of small white dots distributes evenly in the entire growth substrate with the reaction time of $30 \mathrm{~s}$, regardless of exposed periodic mica arrays and modified $\mathrm{NaCl}$ surface as shown in Figure 4b. Increasing reaction time to a

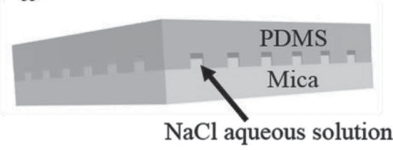

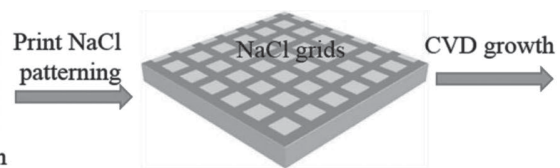

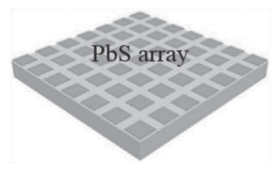

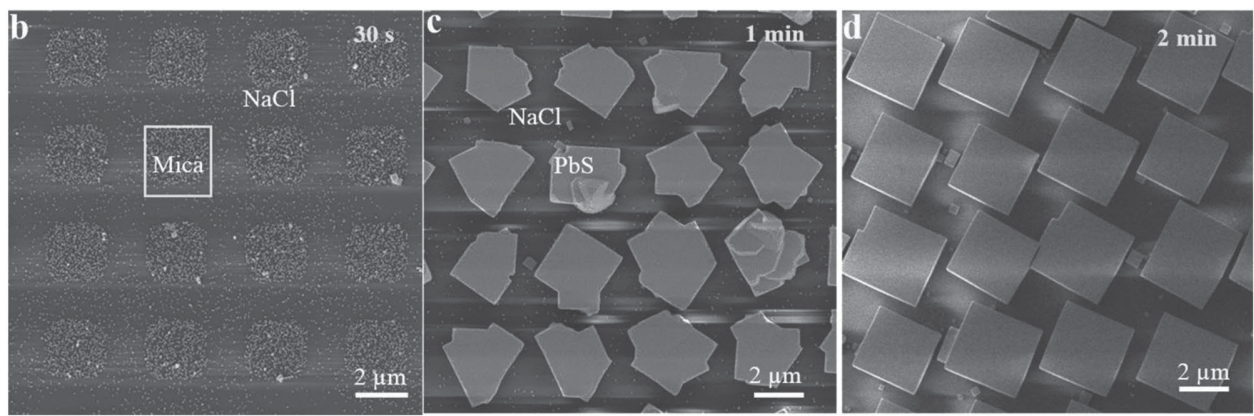

Figure 4. The growing mechanism of 2D PbS nanoplates arrays. a) Schematic representation of procedures for patterning of 2D PbS nanoplates arrays. The SEM images of the PbS nanoplates arrays under different reaction time b) $30 \mathrm{~s}, \mathrm{c}) 1 \mathrm{~min}$, d) $2 \mathrm{~min}$. 
$1 \mathrm{~min}$, plenty of irregular nanoplatelets can be observed and tend to coalesce with each other, as shown in Figure $4 \mathrm{c}$ and Figure S6 (Supporting Information). The driving force of the coalescence process is the reduction of edge energy through decreasing their total edge length. ${ }^{[20]}$ Finally, when the reaction time extends to $2 \mathrm{~min}$, regular square nanoplates appear with final exposed four equivalent $\{200\}$ crystal surfaces. Remarkable, the growing mechanism in our case is different with previous reports about controlling the nucleation site. ${ }^{[42]}$ As shown in Figure 4c,d, only very small $\mathrm{PbS}$ nanoplates can be find on NaCl-modified surface. It further demonstrates that the NaCl-modified surface of mica is highly rough, which hinders the vdWE of PbS.

In summary, by utilizing the nature of low surface energy of graphite, we successfully conducted the van der Waals epitaxial growth of ultrathin 2D PbS nanoplates with thickness of ranging from 5 to $35 \mathrm{~nm}$. The $\mathrm{PbS}$ nanoplates are single crystalline with cubic symmetry and display superior infrared response with photoresponsivity, detectivity, switching time, and photogain values as high as $1621 \mathrm{~A} \mathrm{~W}^{-1}, 1.72 \times 10^{11}$ Jones, $0.3 \mathrm{~s}$, and 2512 respectively. Further considering the epitaxial relation between layered mica and $\mathrm{PbS}$ and using $\mathrm{NaCl}$ to define the position of vdWE, we realize the orientation-controlled and well-defined single-crystalline square $\mathrm{PbS}$ nanopates arrays on mica. The edges of square nanoplates orient predominantly at multiples of $90^{\circ}$. The square $\mathrm{PbS}$ nanopates arrays are promising in the application of high-performance integrated optoelectronic devices.

\section{Experimental Section}

The Experimental Details about the Growth of PbS Nanoplates: The graphite was peeled off with the tape by standard mechanically exploitation method. Then graphite was transferred to surface of $\mathrm{SiO}_{2} / \mathrm{Si}$ substrate. Mica was first intaglio printed by $\mathrm{NaCl}$ aqueous solution using a polydimethylsiloxane (PDMS) stamp. Then, mica with $\mathrm{NaCl}$ aqueous solution $\left(0.01 \mathrm{~mol} \mathrm{~L}^{-1}\right)$ was heated to $150^{\circ} \mathrm{C}$ using a heating belt. Finally, the mica surface modified with periodic arrays was released from the PDMS stamp. The substrates and the PbS powder (99.999\%, Alfa Aesar) were placed into CVD furnace. Before the experiments, the quartz tube was evacuated three times by the pump and flushed with argon. Then $\mathrm{H}_{2}$ at a constant flow rate of $20 \mathrm{sccm}$ was supplied to the tube. During the growth, the temperatures of the precursor and substrates were set to $750^{\circ} \mathrm{C}$ and $500{ }^{\circ} \mathrm{C}$, respectively. The reaction continued for $2 \mathrm{~min}$. At last, the furnace naturally cooled to room temperature.

Microstructure Characterization: The morphology was characterized by optical microscopy (Leica DM4000M microscope), field emission scanning electron microscopy (Hitachi S4800), AFM (Dimension 3100). Then the crystal structure was analyzed by high-resolution transmission electron microscope (HRTEM) on FEI Tecnai F20 with selected area electron diffraction (SAED).

Device Fabrication and Measurements: PbS nanoplates were transferred onto clean $\mathrm{SiO}_{2} / \mathrm{Si}$ substrate from mica by a flexible polydimethylsiloxane(PDMS) layer. The source and drain electrodes were written on PbS nanoplate by standard electron-beam lithography (EBL). Subsequently, $5 \mathrm{~nm} \mathrm{Cr}$ and $100 \mathrm{~nm}$ Au were deposited on surface of PbS. The devices were measured in a manual probe station (Everbeing, BD4) with a sealed chamber. The parameters were analyzed using a Keithley 4200 semiconductor characterization system. All the measurements were carried out in a vacuum of $\approx 10^{-6}$ torr at room temperature. The source and drain electrodes of PbS arrays were prepared by FEI Nanolab 600i SEM/FIB dual-beam system.

\section{Supporting Information}

Supporting Information is available from the Wiley Online Library or from the author.

\section{Acknowledgements}

Y.W. and Q.W. contributed equally to the work. This work was supported by the National Natural Science Foundation of China (Nos. 21373065, 61474033, and 61574050), Strategic Priority Research Program of the Chinese Academy of Sciences (Grant No. XDA09040201), 973 Program of the Ministry of Science and Technology of China (No. 2012CB934103), and CAS Key Laboratory of Nanosystem and Hierarchical Fabrication. The authors also gratefully acknowledge the support of Youth Innovation Promotion Association CAS.

Received: May 10, 2016

Revised: June 2, 2016

Published online:

[1] K. S. Novoselov, A. K. Geim, S. V. Morozov, D. Jiang, Y. Zhang, S. V. Dubonos, I. V. Grigorieva, A. A. Firsov, Science 2004, 306, 666.

[2] S. Najmaei, Z. Liu, W. Zhou, X. Zou, G. Shi, S. Lei, B. I. Yakobson, J.-C. Idrobo, P. M. Ajayan, J. Lou, Nat. Mater. 2013, 12, 754.

[3] H. Li, J. Cao, W. Zheng, Y. Chen, D. Wu, W. Dang, K. Wang, H. Peng, Z. Liu, J. Am. Chem. Soc. 2012, 134, 6132.

[4] X. Wang, Y. Ouyang, X. Li, H. Wang, J. Guo, H. Dai, Phys. Rev. Lett. 2008, 100, 206803.

[5] a) K. S. Novoselov, A. K. Geim, S. V. Morozov, D. Jiang, M. I. Katsnelson, I. V. Grigorieva, S. V. Dubonos, A. A. Firsov, Nature 2005, 438, 197; b) Y. Zhang, Y.-W. Tan, H. L. Stormer, P. Kim, Nature 2005, 438, 201.

[6] K. Takada, H. Sakurai, E. Takayama-Muromachi, F. Izumi, R. A. Dilanian, T. Sasaki, Nature 2003, 422, 53.

[7] C.-Z. Chang, J. Zhang, X. Feng, J. Shen, Z. Zhang, M. Guo, K. Li, Y. Ou, P. Wei, L.-L. Wang, Z.-Q. Ji, Y. Feng, S. Ji, X. Chen, J. Jia, X. Dai, Z. Fang, S.-C. Zhang, K. He, Y. Wang, L. Lu, X.-C. Ma, Q.-K. Xue, Science 2013, 340, 167.

[8] A. A. Balandin, Nat. Mater. 2011, 10, 569.

[9] a) C. Zhu, Z. Zeng, H. Li, F. Li, C. Fan, H. Zhang, J. Am. Chem. Soc. 2013, 135, 5998; b) F. K. Perkins, A. L. Friedman, E. Cobas, P. M. Campbell, G. G. Jernigan, B. T. Jonker, Nano Lett. 2013, 13, 668.

[10] a) D. J. Late, B. Liu, J. Luo, A. Yan, H. S. S. R. Matte, M. Grayson, C. N. R. Rao, V. P. Dravid, Adv. Mater. 2012, 24, 3549; b) C. R. Dean, A. F. Young, I. Meric, C. Lee, L. Wang, S. Sorgenfrei, K. Watanabe, T. Taniguchi, P. Kim, K. L. Shepard, J. Hone, Nat. Nano 2010, 5, 722.

[11] L. Cheng, J. Liu, X. Gu, H. Gong, X. Shi, T. Liu, C. Wang, X. Wang, G. Liu, H. Xing, W. Bu, B. Sun, Z. Liu, Adv. Mater. 2014, 26, 1886.

[12] S. Lei, L. Ge, Z. Liu, S. Najmaei, G. Shi, G. You, J. Lou, R. Vajtai, P. M. Ajayan, Nano Lett. 2013, 13, 2777.

[13] X.-J. Wu, J. Chen, C. Tan, Y. Zhu, Y. Han, H. Zhang, Nat. Chem. 2016, 8, 470

[14] Y. Liu, X. Hua, C. Xiao, T. Zhou, P. Huang, Z. Guo, B. Pan, Y. Xie, J. Am. Chem. Soc. 2016, 138, 5087.

[15] A. L. Briseno, S. C. B. Mannsfeld, M. M. Ling, S. Liu, R. J. Tseng, C. Reese, M. E. Roberts, Y. Yang, F. Wudl, Z. Bao, Nature 2006, 444, 913.

[16] Y. Zhou, Y. Nie, Y. Liu, K. Yan, J. Hong, C. Jin, Y. Zhou, J. Yin, Z. Liu, H. Peng, ACS Nano 2014, 8, 1485. 
[17] Y. Guo, M. Aisijiang, K. Zhang, W. Jiang, Y. Chen, W. Zheng, Z. Song, J. Cao, Z. Liu, H. Peng, Adv. Mater. 2013, 25, 5959.

[18] W. Zheng, T. Xie, Y. Zhou, Y. L. Chen, W. Jiang, S. Zhao, J. Wu, Y. Jing, Y. Wu, G. Chen, Y. Guo, J. Yin, S. Huang, H. Q. Xu, Z. Liu, H. Peng, Nat. Commun. 2015, 6, 6972.

[19] G. Wang, D. Li, H.-C. Cheng, Y. Li, C.-Y. Chen, A. Yin, Z. Zhao, Z. Lin, H. Wu, Q. He, M. Ding, Y. Liu, Y. Huang, X. Duan, Sci. Adv. 2015, 1, 1500613

[20] L. Niu, X. Liu, C. Cong, C. Wu, D. Wu, T. R. Chang, H. Wang, Q. Zeng, J. Zhou, X. Wang, W. Fu, P. Yu, Q. Fu, S. Najmaei, Z. Zhang, B. I. Yakobson, B. K. Tay, W. Zhou, H. T. Jeng, H. Lin, T. C. Sum, C. Jin, H. He, T. Yu, Z. Liu, Adv. Mater. 2015, 27, 7800.

[21] Q. Wang, M. Safdar, K. Xu, M. Mirza, Z. Wang, J. He, ACS Nano 2014, 8, 7497

[22] Q. Wang, K. Xu, Z. Wang, F. Wang, Y. Huang, M. Safdar, X. Zhan, F. Wang, Z. Cheng, J. He, Nano Lett. 2015, 15, 1183.

[23] Q. Wang, Y. Wen, F. Yao, Y. Huang, Z. Wang, M. Li, X. Zhan, K. Xu, F. Wang, F. Wang, J. Li, K. Liu, C. Jiang, F. Liu, J. He, Small 2015, 11, 5388.

[24] G. Konstantatos, I. Howard, A. Fischer, S. Hoogland, J. Clifford, E. Klem, L. Levina, E. H. Sargent, Nature 2006, 442, 180.

[25] A. Rogalski, Prog. Quantum Electron. 2003, 27, 59.

[26] a) P. L. Nichols, Z. Liu, L. Yin, S. Turkdogan, F. Fan, C. Z. Ning, Nano Lett. 2015, 15, 909; b) E. M. Miller, D. M. Kroupa, J. Zhang P. Schulz, A. R. Marshall, A. Kahn, S. Lany, J. M. Luther, M. C. Beard, C. L. Perkins, J. van de Lagemaat, ACS Nano 2016, 10, 3302.

[27] a) R. Schodel, T. Ott, R. Genzel, R. Hofmann, M. Lehnert, A. Eckart, N. Mouawad, T. Alexander, M. J. Reid, R. Lenzen, M. Hartung, F. Lacombe, D. Rouan, E. Gendron, G. Rousset, A. M. Lagrange, W. Brandner, N. Ageorges, C. Lidman, A. F. M. Moorwood, J. Spyromilio, N. Hubin, K. M. Menten, Nature 2002, 419, 694 b) S. Rawlings, M. Lacy, K. M. Blundell, S. A. Eales, A. J. Bunker, S. T. Garrington, Nature 1996, 383, 502; c) R. Ettl, I. Chao, F. Diederich, R. L. Whetten, Nature 1991, 353, 149.

[28] S. A. McDonald, G. Konstantatos, S. Zhang, P. W. Cyr, E. J. D. Klem, L. Levina, E. H. Sargent, Nat. Mater. 2005, 4, 138.
[29] X.-B. Li, P. Guo, Y.-N. Zhang, R.-F. Peng, H. Zhang, L.-M. Liu, J. Mater. Chem. C 2015, 3, 6284

[30] M. Aerts, T. Bielewicz, C. Klinke, F. C. Grozema, A. J. Houtepen, J. M. Schins, L. D. A. Siebbeles, Nat. Commun. 2014, 5, 3789.

[31] G. Konstantatos, M. Badioli, L. Gaudreau, J. Osmond, M. Bernechea, F. P. G. de Arquer, F. Gatti, F. H. L. Koppens, Nat. Nano 2012, 7, 363.

[32] Z. Sun, Z. Liu, J. Li, G.-a. Tai, S.-P. Lau, F. Yan, Adv. Mater. 2012, 24 5878.

[33] D. Kufer, I. Nikitskiy, T. Lasanta, G. Navickaite, F. H. L. Koppens, G. Konstantatos, Adv. Mater. 2015, 27, 176.

[34] W. Zheng, W. Feng, X. Zhang, X. Chen, G. Liu, Y. Qiu, T. Hasan, P. Tan, P. A. Hu, Adv. Funct. Mater. 2016, 26, 2648.

[35] J. Schornbaum, B. Winter, S. P. Schießl, F. Gannott, G. Katsukis, D. M. Guldi, E. Spiecker, J. Zaumseil, Adv. Funct. Mater. 2014, 24, 5798.

[36] H. Heo, J. H. Sung, G. Jin, J.-H. Ahn, K. Kim, M.-J. Lee, S. Cha, H. Choi, M.-H. Jo, Adv. Mater. 2015, 27, 3803.

[37] Y. Hernandez, V. Nicolosi, M. Lotya, F. M. Blighe, Z. Sun, S. De, I. T. McGovern, B. Holland, M. Byrne, Y. K. Gun'Ko, J. J. Boland, P. Niraj, G. Duesberg, S. Krishnamurthy, R. Goodhue, J. Hutchison, V. Scardaci, A. C. Ferrari, J. N. Coleman, Nat. Nano 2008, 3, 563.

[38] Z. Wang, K. Xu, Y. Li, X. Zhan, M. Safdar, Q. Wang, F. Wang, J. He, ACS Nano 2014, 8, 4859.

[39] F. Wang, Z. Wang, K. Xu, F. Wang, Q. Wang, Y. Huang, L. Yin, J. He, Nano Lett. 2015, 15, 7558

[40] Q. Wang, J. Li, Y. Lei, Y. Wen, Z. Wang, X. Zhan, F. Wang, F. Wang, Y. Huang, K. Xu, J. He, Adv. Mater. 2016, 28, 3596.

[41] Y. Guo, Z. Liu, H. Peng, Small 2015, 11, 3290

[42] a) G. H. Han, N. J. Kybert, C. H. Naylor, B. S. Lee, J. Ping, J. H. Park, J. Kang, S. Y. Lee, Y. H. Lee, R. Agarwal, A. T. C. Johnson, Nat. Commun. 2015, 6, 6128; b) Q. Yu, L. A. Jauregui, W. Wu, R. Colby, J. Tian, Z. Su, H. Cao, Z. Liu, D. Pandey, D. Wei, T. F. Chung, P. Peng, N. P. Guisinger, E. A. Stach, J. Bao, S.-S. Pei, Y. P. Chen, Nat. Mater. 2011, 10, 443; c) G. Su, V. G. Hadjiev, P. E. Loya, J. Zhang, S. Lei, S. Maharjan, P. Dong, P. M. Ajayan, J. Lou, H. Peng, Nano Lett. 2015, 15, 506. 